\begin{tabular}{|c|l|}
\hline Title & Controlling Engine System : a Low-Dimensional Dynamics in a Spark Ignition Engine of a Motorcycle \\
\hline Author(s) & Matsumoto, Kazuhiro; Tsuda, Ichiro; Hosoi, Y uki \\
\hline Citation & Zeitschrift für Naturforschung A, 62a, 587-595 \\
\hline Issue Date & 2007 \\
\hline Doc URL & http://hdl.handle.net/2115/30226 \\
\hline Type & article \\
\hline File Information & ZNA 62A .pdf \\
\hline
\end{tabular}

Instructions for use 


\title{
Controlling Engine System: a Low-Dimensional Dynamics in a Spark Ignition Engine of a Motorcycle
}

\author{
Kazuhiro Matsumoto ${ }^{\mathrm{a}}$, Ichiro Tsuda ${ }^{\mathrm{a}, \mathrm{b}}$, and Yuki Hosoi ${ }^{\mathrm{c}}$ \\ a Hokkaido University, Department of Mathematics, Kita 10 Nishi 8, Kita-ku, \\ Sapporo 060-0810, Japan \\ ${ }^{\mathrm{b}}$ Hokkaido University, Research Institute for Electronic Science, Kita 12 Nishi 6, Kita-ku, \\ Sapporo 060-0812, Japan \\ c Yamaha Motor Co., Ltd., Technical Development Division, 2500 Shingai, Iwata 438-8501, Japan \\ Reprint requests to K. M.; Fax: +81-11-706-2636; E-mail: matsumoto@ math.sci.hokudai.ac.jp \\ Z. Naturforsch. 62a, 587 -595 (2007); received May 29, 2007
}

\begin{abstract}
We analyze a time series of the combustion pressure in the idle state, measured from a spark ignition engine of a motorcycle. It is clarified that the engine system can be described by a lowdimensional deterministic dynamics perturbed by some stochastic process. We also propose a method to stabilize the chaotic behaviour of engine's data by adopting the Pyragas' method. We actually use this method in a computer experiment for the control of combustion pressure data to demonstrate the efficiency of the proposed method. As a result of the experiment, we eliminate the fluctuations in the combustion pressure data and obtain a periodic orbit.
\end{abstract}

Key words: Nonlinear Time Series Analysis; Attractor Reconstruction; Combustion; Controlling Chaos; Delayed Feedback Control.

\section{Introduction}

Cycle-to-cycle combustion variations in spark ignition engines have been a subject of intensive research for many years. Instabilities of the combustion process, which result in the difficulty of controlling engines, can be a cause of harmful fluctuations of the power output, which results in the difficulty of controlling engines [1-3]. It is known that elimination of fluctuations of cyclic motion would lead to a $10 \%$ increase in the power output of engines $[4,5]$. It is thus of great importance to control the motion of engines by eliminating the fluctuations, which leads to an improvement of engine performance.

The main sources of combustion instabilities are fluctuations in the gaseous motion inside the cylinder during combustion, fluctuations in the amount of fuel, air and recycled exhaust-gas supplied to the cylinder, and fluctuations in the mixing of fresh mixture and residual gas inside the cylinder near the spark plug [6]. It has been discussed whether these factors stem from a stochastic or deterministic process. Kantor [7] conducted a study of spark ignition engines by using his exhaust-gas temperature model. Daw et al. $[8,9]$ proposed a model of gas circulation, which combines stochastic and nonlinear deterministic elements. Recently several researchers analyzed internal combustion pressure data in terms of nonlinear dynamics $[1,5]$. The results suggest that the engine systems have a nonlinear dynamical structure, although the data include stochastic parts.

Motivated by the above facts, we also analyzed several kinds of spark ignition engine data of a motorcycle, which were obtained under the collaboration of the Yamaha Motor Co., Ltd. The experiments were conducted under two conditions of engine revolutions: one at 1,400 rpm without load, which corresponds to the idle state, and the other at 4,350 rpm with a steady load (see Section 2 for more details). Even though the external input, which drives the engine to run, is periodic, the amplitude in the pressure data is fluctuated under both conditions. At first, the fluctuations were considered due to stochastic noise such as thermal noise. It was, however, clarified from the results of the analysis, that the engine system has a low-dimensional deterministic dynamics. The presence of the deterministic components of the engine system means that it is possible to control the motion of the engine's output by utilizing the underlying dynamics, in principle. We therefore considered the control method to eliminate the fluctuations. In the scheme of controlling chaos, we propose a control method of the engine system by adopting the delayed feedback control [10]. 
The characteristic of Pyragas' method is that, even if we do not know the equations of the dynamical system concerned, we can obtain a periodic orbit simply by using its time series and its delayed feedback. We do not require to take a Poincaré section. It is therefore easily applied to experimental systems. As for the subject of controlling chaos, the Ott-Grebogi-Yorke (OGY) method [11] is also popular and useful. In the OGY method we can only obtain the unstable periodic orbits embedded in chaotic attractors, because the underlying dynamics is not essentially changed. In contrast, Pyragas' method essentially changes the underlying dynamics with a delayed feedback term. If the delayed feedback term converges, we can obtain an unstable periodic orbit embedded in the chaotic attractor; but otherwise a new different solution from that of the original dynamical system is created. It is known that this method can stabilize chaotic dynamics which has the Hopf bifurcation or period-doubling bifurcation, although it fails to stabilize the saddle-node type of chaotic dynamics that the OGY method can do [12]. Furthermore, Pyragas' method cannot specify the desired orbit which is stabilized even if the delayed feedback term converges, but the OGY method can do the desired orbit strictly if one can find the unstable periodic orbit in phase space with a Poincaré section. However, it is normally difficult to find unstable periodic orbits in the online process of application of the OGY method.

As stated above, each method has its drawbacks and advantages. Our purpose was to eliminate the fluctuations in the engine system and to obtain a periodic orbit. We thus did not adhere to the form of the obtained target orbit and just only had to make the motion of the engine's output periodic. We utilized Pyragas' method considering how this method is effective on the engine system.

The organization of the paper is as follows. The experimental data are provided in Section 2. In Section 3, we present the results of the nonlinear analysis for internal combustion pressure data in the idle state. We propose a control method and demonstrate the efficiency of the method with a computer experiment in Section 4. Section 5 is devoted to conclusions and discussion.

\section{Experimental Data}

The experiments were carried out with a fourstroke [13], single-cylinder $250 \mathrm{cc}$ engine at Yamaha
Motor Co., Ltd. The experimental data were taken under two conditions of engine revolutions: one at $1,400 \mathrm{rpm}$ without load, which corresponds to the idle state, and the other at 4,350 rpm with a steady load. Each data point was measured consecutively against a crank angle for 1,000 cycles. The sampling times were approximately $8.4 \mathrm{kHz}$ for the case of 1,400 rpm, and approximately $26.1 \mathrm{kHz}$ for $4,350 \mathrm{rpm}$, respectively. The cycle here means the combustion cycle. Each combustion (reciprocating movement of the four strokes) is performed in two revolutions of the engine. In terms of the crank angle, one cycle means 720 degrees, consisting of 720 points.

Five kinds of data were obtained for each of the two conditions: pressure (internal combustion pressure in the cylinder), boost voltage (related to the amount of air intake), hydrocarbon (exhaust-gas, related to the amount of residual gas), air-to-fuel ratio and injection current (related to the amount of fuel injection). The injection current that determines the amount of fuel injection has a pulse shape. It is considered to be the external input for the engine system. The interval of pulses is almost constant. The air-to-fuel ratio should theoretically be a constant, although the value varies slightly during the experiment. It can be regarded as a control parameter. We focused on the three kinds of data, the combustion pressure, the boost voltage and the hydrocarbon, and analyzed them. These data are not periodic, while the external input (the injection current) is almost periodic. Especially, the fluctuations of the low-frequency components at the lower revolution $(1,400 \mathrm{rpm})$ are much larger than those at the higher revolution $(4,350 \mathrm{rpm})$. The dynamics of the engine may be stabilized at the higher revolution by the suppression of the fluctuations of low-frequency components. Then only the fluctuations of high-frequency components would be prominent, whose components cannot be distinguished from thermal noise. Therefore it is particularly valuable to analyze the idle state $(1,400 \mathrm{rpm})$. In the following section, we will show the analysis of the pressure data in the idle state.

\section{Time Series Analysis for Combustion Pressure Data in the Idle State}

Figure 1 shows a typical time series of the combustion pressure represented by the crank angle for $15 \mathrm{cy}-$ cles. It was directly measured from a pressure sensor inside the cylinder and thus contains noise such as electrical noise. First of all, we reconstruct the at- 

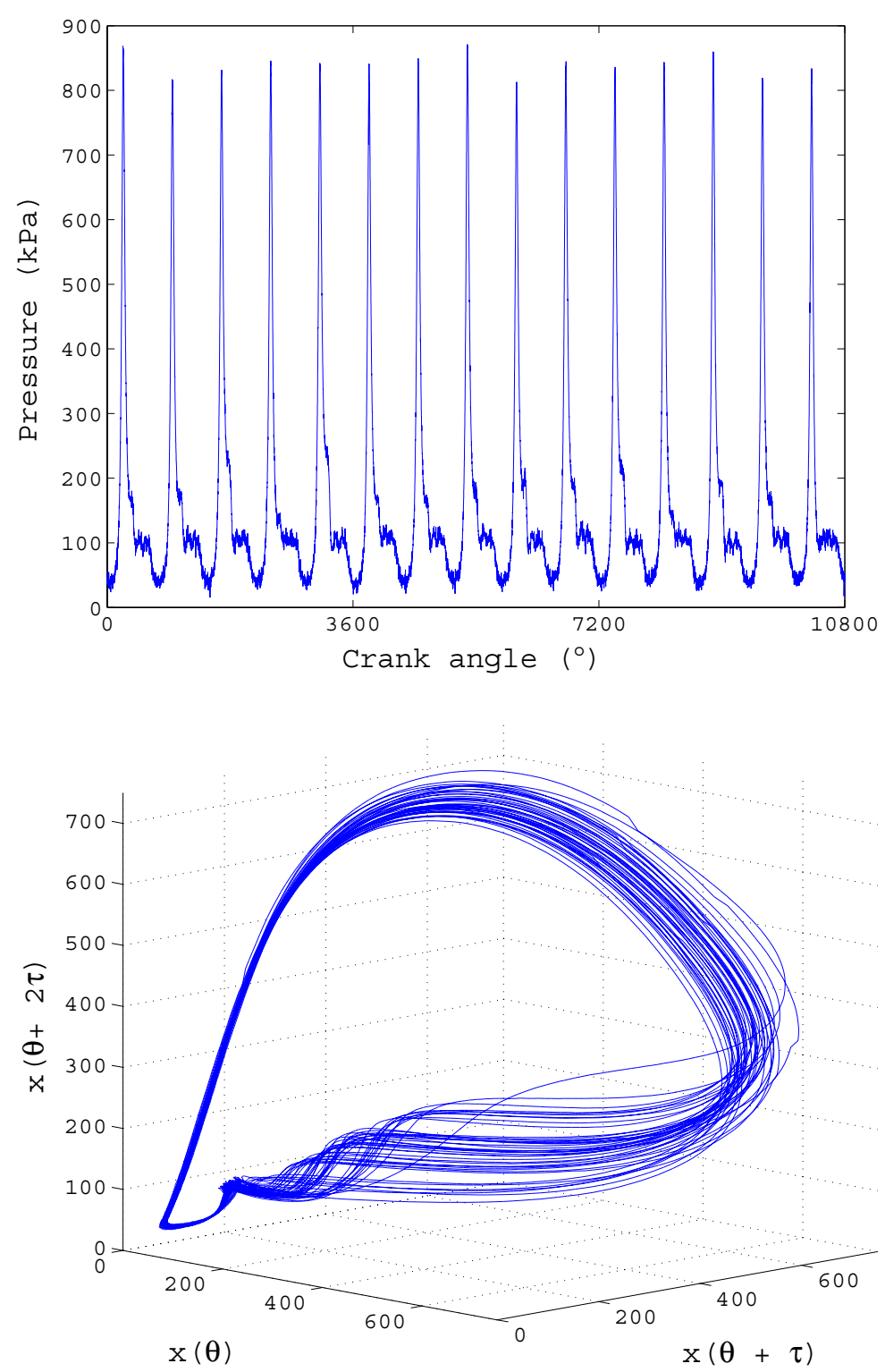

Fig. 1. Typical time series of the combustion pressure of 15 cycles.

Fig. 2. Reconstructed attractor projected to the three-dimensional space of the pressure data. About 50 cycles are depicted for visibility. tractor from the pressure data by applying the embedding technique $[14,15]$. Applying a method of moving average to the original data of Fig. 1, we removed noise slightly for the reconstruction. We used the data after such a noise reduction. We denoted a crank angle by $\theta$ [strictly speaking, the crank angle is not constant in time, so that it should be denoted by $\theta(t)$, but the crank angle can be regarded as an independent variable, since the fluctuation is comparatively small]. Then, a time series of the pressure was denoted by a variable $x(\theta)$. Given a delay time $\tau$ and an em- bedding dimension $m$, we could make an embedding vector $\boldsymbol{x}(\theta)=(x(\theta), x(\theta+\tau), \ldots, x(\theta+(m-1) \tau))$. The delay time was chosen to be $\tau=30$ by trial-anderror. In order to determine the number of the embedding dimension, we adopted the false nearest neighbours method [16]. We estimated $m=4$, designating a percentage of false nearest neighbours below $5 \%$ as the criterion. We show the reconstructed attractor projected to the three-dimensional space in Fig. 2, where about 50 cycles are depicted for visibility. From Figs. 1 and 2 , one can see that the amplitude fluctuates. Al- 

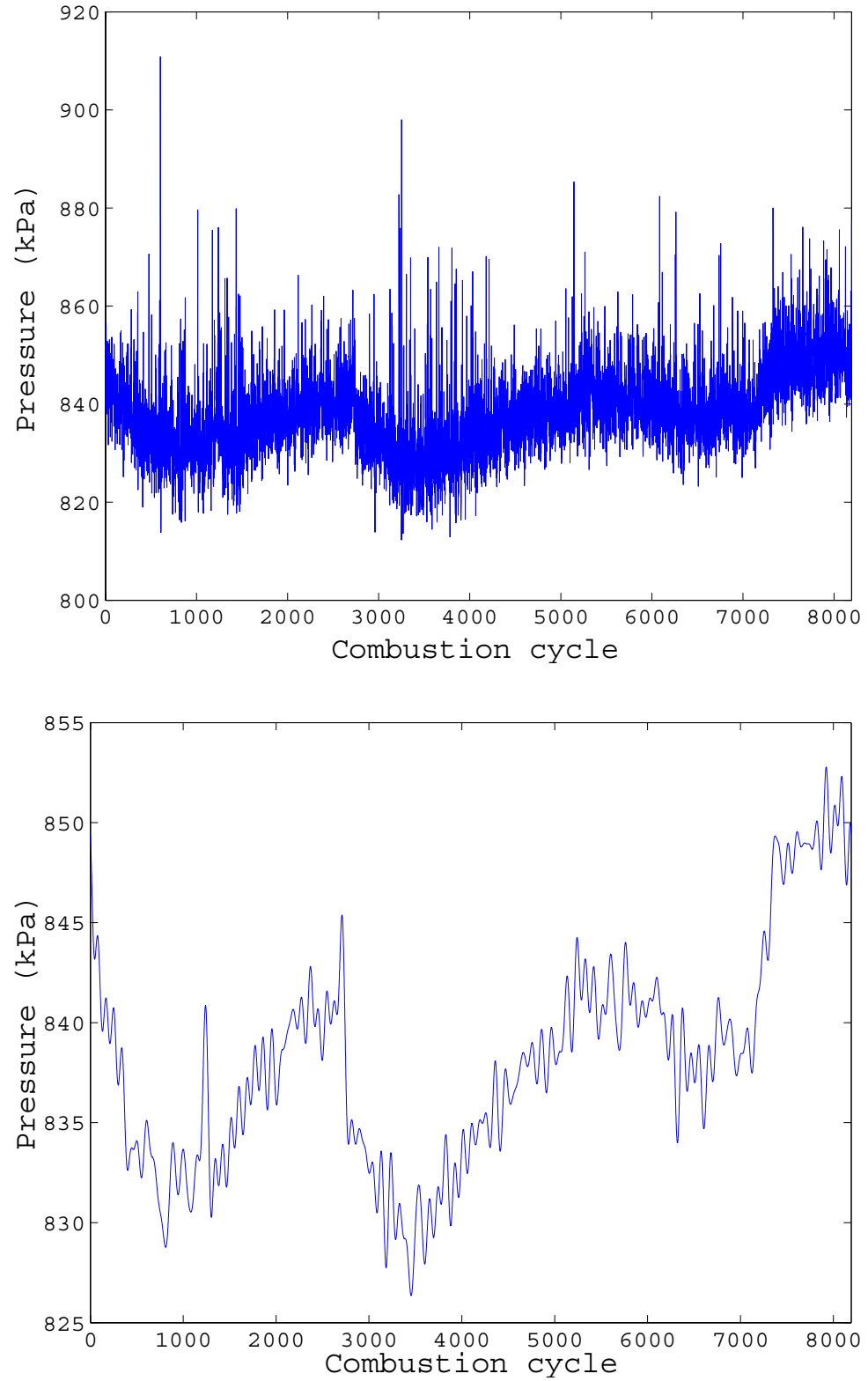

Fig. 3. Local maxima of the pressure data vs. the combustion cycle.
Fig. 4. Data of the low-frequency components of Figure 3. though the external input is periodic, the behaviour of the pressure is not totally periodic. If a stable dynamics exists in this engine system, the orbit of the pressure would become a limit cycle. There is, however, some deviation from a limit cycle. We can consider two possibilities of the reason: this deviation is caused by a destabilization of the dynamics or just by a stochastic process.

In order to study the reason, we plotted the local maxima of the pressure data. The data of 1,000 cy- cles were not sufficient for this purpose. We therefore used the local maximum data for 10,000 cycles measured in another trial but under the same conditions. The data are shown for 8,192 cycles in Figure 3. The long-term fluctuations are clearly observed. It is conceivable that the low-frequency components result from a certain dynamics. On the other hand, the high-frequency components are probably due to noise. We examined the two parts separately. 

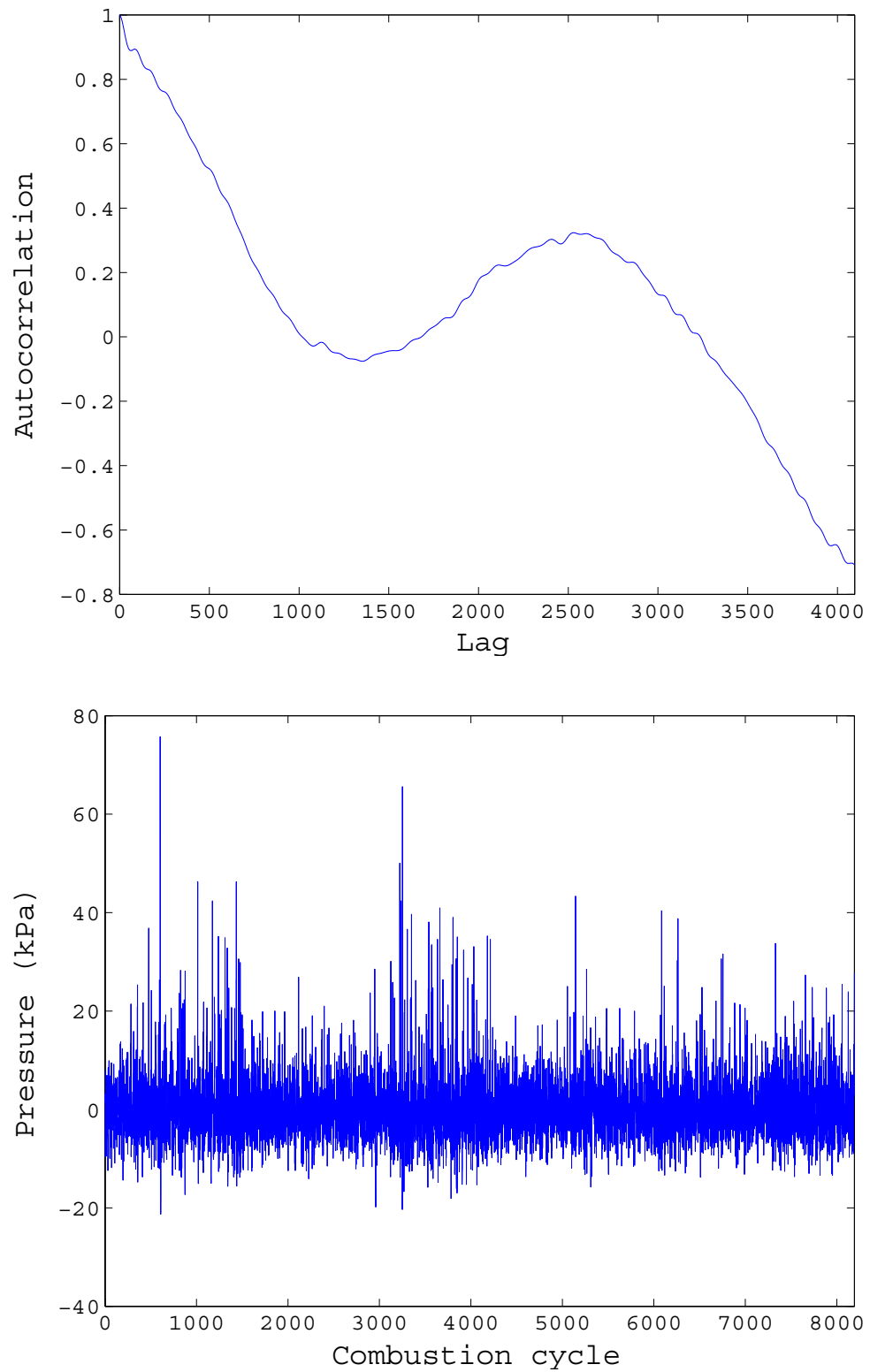

Fig. 5. Autocorrelation coefficients for the pressure data of the low-frequency components.

Fig. 6. Data of the high-frequency components of Figure 3.
Figure 4 shows the data of the low-frequency components obtained by the use of a low-pass filter. We calculated autocorrelation coefficients for the data, which are shown in Figure 5. We can see that there exist periodic components of 2,500 to 3,000 cycles. This suggests that a slow deterministic dynamics underlies the data. We also calculated the Lyapunov exponents, using the Sano-Sawada method [17]. The embedding dimension was $m=3$, because the operation of taking local maxima is equivalent to taking a Poincaré section; thus one dimension was reduced. The delay time was determined to be $\tau=1,000$ by trial-anderror. The result was $\lambda_{1}=0.66, \lambda_{2}=-0.12$ and $\lambda_{3}=$ -1.11 . This result suggests that the engine system produces chaos, because there is at least one positive Lyapunov exponent. For comparison, we show the result of $m=2$. In this case, we obtained $\lambda_{1}=0.29$ and $\lambda_{2}=$ -1.10 . We calculated the Lyapunov dimensions for both cases $m=2$ and $m=3$, which are denoted by $D_{2 \mathrm{~L}}$ and $D_{3 \mathrm{~L}}$, respectively: $D_{2 \mathrm{~L}}=1+0.29 /|-1.10| \approx 1.26$ 


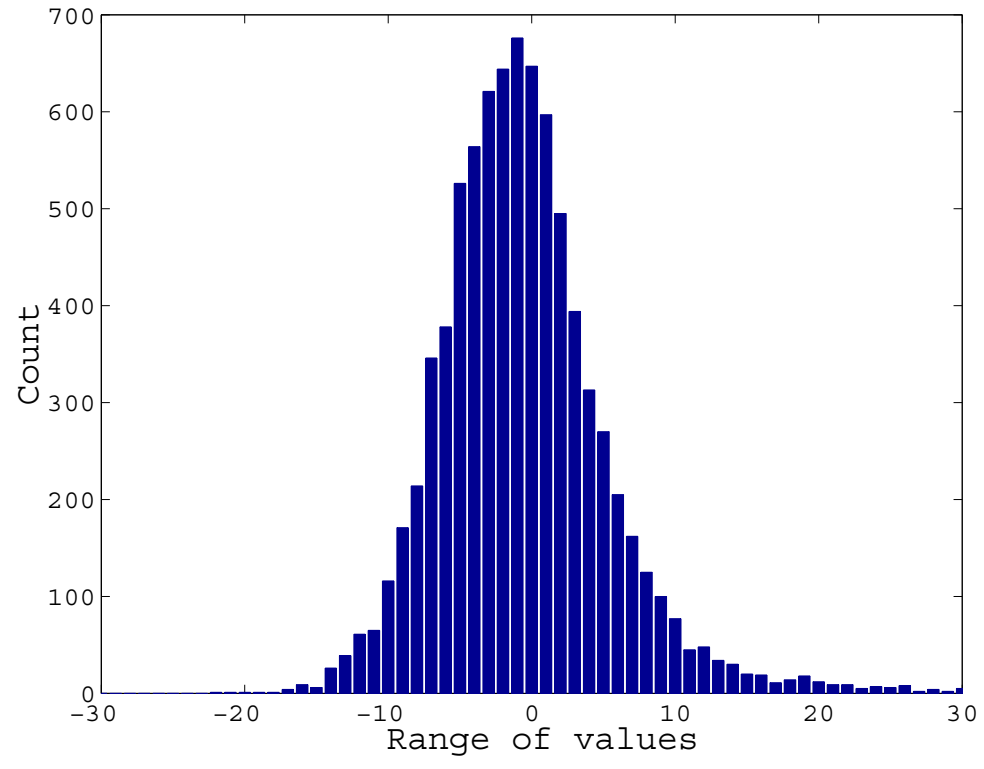

Fig. 7. Histogram of the fluctuations of the high-frequency components. and $D_{3 \mathrm{~L}}=2+(0.66-0.12) /|-1.11| \approx 2.55$. These are the results in the discrete system of taking the Poincaré section. Returning to the continuous system before taking the Poincaré section, the Lyapunov dimensions $D_{2 \mathrm{~L}}$ and $D_{3 \mathrm{~L}}$ become 2.26 and 3.55 , respectively. Judging from these results, one might consider that the reconstructed attractor shown in Fig. 2 can be embedded in three dimensions. If this case should be true, $\lambda_{2}=-0.12$ estimated at $m=3$ in the discrete system would be spurious, but in this paper we followed the criterion of a percentage of false nearest neighbours below $5 \%$, as we already mentioned, and adopted the embedding dimension $m=4$ in the continuous system. We, in turn, adopted the embedding dimension $m=3$ in the discrete system after taking the Poincaré section.

Next, we focused on the fluctuations of highfrequency components. Figure 6 shows the data of the high-frequency components, which are obtained by subtracting the data shown in Fig. 4 from the data shown in Figure 3. We divided the range of values in Fig. 6 into 160 bins to count points which fall into each bin, and then obtained the histogram shown in Fig. 7, where it is depicted in the range of $[-30: 30]$ for visibility. As can be seen in this figure, the distribution is approximated by the Gaussian distribution. Our speculation is plausible that the highfrequency components result from a stochastic process. One might consider that the behaviour including the high-frequency components in the engine sys- tem stems from a high-dimensional chaos or even an infinite-dimensional chaos which is described by a partial differential equation. However, new dynamical behaviours can essentially appear in reduction systems whose dimension is lower than that of the original systems [18]. In the case of the engine system, the new dynamics in the reduction system corresponds to a lowdimensional dynamics consisting of the low-frequency components. Based on this standpoint, it is legitimate to consider that the remaining part consisting of the high-frequency components results from a stochastic process.

In conclusion, it was clarified that the engine system shows a low-dimensional dynamics which is probably chaos. In the next section we consider a control method to eliminate the fluctuations of the time series as seen in Figure 1.

\section{A New Method for Controlling the Engine System}

In this section, we consider a way of controlling the engine system within the framework of controlling chaos. We have two approaches to control the engine system: One is to clarify the underlying dynamics in the engine system and then control it, and the other is to directly control it without clarifying the dynamics. We consider the latter approach and propose a method to stabilize the deterministic but chaotic behaviour in the 


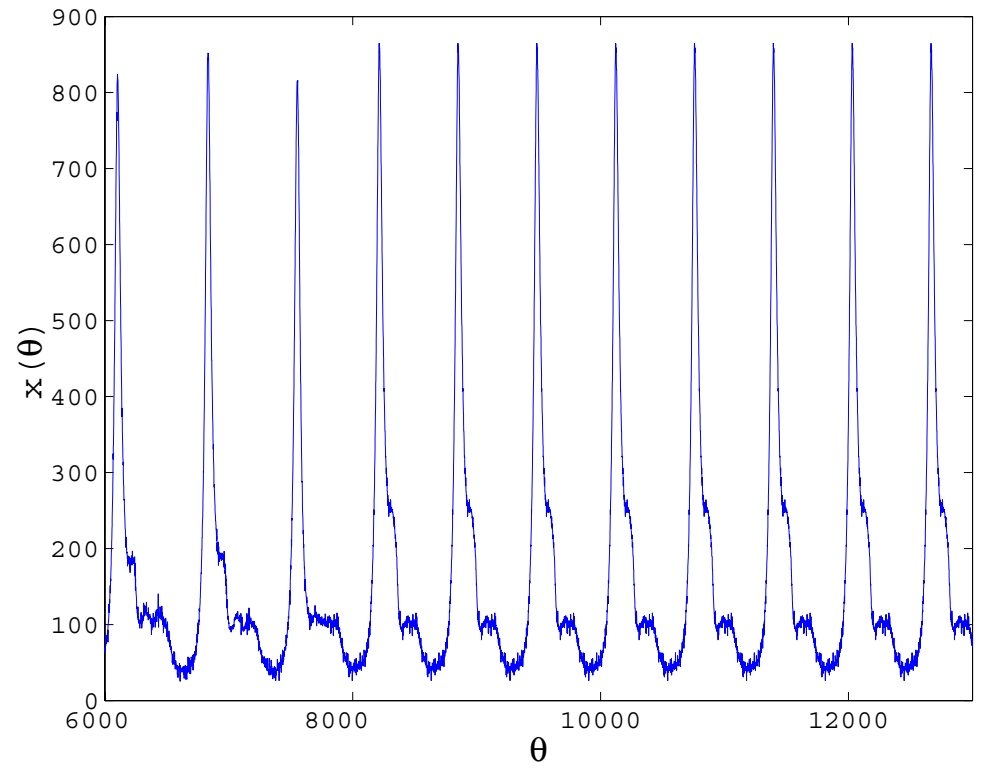

Fig. 8. Application of the method proposed here to the pressure data. A period-one orbit was obtained by the effect of feedback control. engine's data, applying Pyragas' method. As already mentioned in Section 1, this method is easily applicable to experimental systems. Examples of experimental implementation are electronic chaos oscillators [19], magnet-elastic systems [20], lasers [21] and a cardiac system [22].

Our idea is as follows. As already mentioned in Section 3 , the engine system includes a low-dimensional dynamics. We can describe the dynamics, the formula of which is actually unknown, as

$$
\dot{\boldsymbol{X}}(\boldsymbol{\theta})=\boldsymbol{F}(\boldsymbol{X}(\boldsymbol{\theta})),
$$

where time is represented by the crank angle $\theta$ as in Section 3 and $\boldsymbol{X}=\left(x_{1}, x_{2}, \ldots, x_{m}\right)$ is a state vector. From the results of Section 3, the time series of the combustion pressure mainly follows a dynamical system, and is thus represented by a dynamical variable denoted by $x_{1}(\theta)$. In order to apply Pyragas' method, we observe the pressure $x_{1}(\theta)$ and add the delayed feedback $D(\theta)=x_{1}(\theta-T)-x_{1}(\theta)$ with the delay time $T$, which is the period of a desired orbit, to the engine system:

$$
\dot{\boldsymbol{X}}(\theta)=\boldsymbol{F}(\boldsymbol{X}(\theta))+G(\theta) \boldsymbol{C},
$$

where

$$
G(\theta)= \begin{cases}-F_{0}, & \text { if } K D(\theta) \leq-F_{0}, \\ K D(\theta), & \text { if }-F_{0}<K D(\theta)<F_{0}, \\ F_{0}, & \text { if } K D(\theta) \geq F_{0},\end{cases}
$$

and $\boldsymbol{C}$ is the column vector: $c_{11}=1$ and $c_{j 1}=0$ for $2 \leq$ $j \leq m$. Here $K$ is a feedback gain, $F_{0}>0$ is a saturating value of the feedback and $T$ becomes 720 since we want to make one combustion cycle be exactly two revolutions of the engine. There is a reason for placing the saturating value $F_{0}$. Applying the present method directly to actual engines, the large perturbation can cause abnormal combustion and lead to engine stall. The saturating value prevents this phenomenon.

In order to examine the method, we performed a computer experiment for the combustion pressure data. Actually, we used the embedding technique since we did not explicitly have all of the dynamical variables in the engine system. In Section 3, the delay time and the embedding dimension of time series of combustion pressure were determined to be $\tau=30$ and $m=4$, respectively. We consider the following type of system:

$$
\begin{aligned}
\dot{x}(\theta) & =f_{1}(x, y, z, w)+G(\theta), \\
\dot{y}(\theta) & =f_{2}(x, y, z, w), \\
\dot{z}(\theta) & =f_{3}(x, y, z, w), \\
\dot{w}(\theta) & =f_{4}(x, y, z, w),
\end{aligned}
$$

where $\mathrm{x}(\theta)=x_{1}(\theta), \mathrm{y}(\theta)=x_{1}(\theta+\tau), \mathrm{z}(\theta)=x_{1}(\theta+$ $2 \tau)$ and $\mathrm{w}(\theta)=x_{1}(\theta+3 \tau)$. Because we cannot define the vector field at all points of phase space in (4), due to a finite number of data, the problem is that a point perturbed by the effect of the feedback does not have any corresponding velocity. Each point in the embed- 
ded orbit has an approximated velocity. Utilizing this information, we replace the perturbed point with the nearest neighbour point in the embedded orbit [23].

The experiment was conducted for the pressure data that contain 1,000 cycles as shown in Fig. 1 with $K=$ 0.1 and $F_{0}=0.4$. The result is illustrated in Figure 8. In this experiment, the feedback control was provided throughout the time course starting at $\theta=0$. A period-one orbit was obtained by the effect of the feedback control, which was established at approximately $8,000^{\circ}$ of the crank angle corresponding to the eleventh combustion cycle. However, this result is not exactly successful. The period of the obtained periodic orbit is not 720 but 636 , which means that the feedback term does not converge. In other words, the resultant solution is not a solution embedded in the original attractor but a new solution created by the effect of the feedback term. The obtained orbit is, however, periodic and the fluctuations are eliminated. This fact motivates us to conduct actual experiments in online control.

\section{Conclusion and Discussion}

We presented the results of time series analysis for the combustion pressure data in the idle state by dividing the local maximum data of the pressure into two parts: the low-frequency components and the high-frequency components. We showed that the low-frequency components are caused by a lowdimensional dynamics which is a slow dynamics with long-term correlations, and the high-frequency components are caused by a stochastic process. In the present framework it is concluded that the cycle-to-cycle combustion variations in engines would result from the interplay of chaotic dynamics of engine systems and a stochastic process. If the chaotic dynamics is dominant in engine systems as compared to the stochastic process, it is possible to control them by utilizing the dynamics.

Based on this idea, we proposed a method for controling the engine by adopting Pyragas' method. In order to examine the method, we made a computer exper-

[1] M. Wendeker, G. Litak, J. Czarnigowski, and K. Szabelski, Int. J. Bifurcation and Chaos 14, 1801 (2004).

[2] G. Litak, T. Kamiński, R. Rusinek, J. Czarnigowski, and M. Wendeker, Chaos, Solitons and Fractals 35, 578 (2008). iment. Since we did not know all of the dynamical variables in the engine system, we used only the pressure data to verify it in a topological sense. We actually stabilized the fluctuations of the pressure data and generated a period-one orbit with the proposed method. The period of a stabilized orbit did not, however, coincide with the period of the engine cycle. Two possible reasons can be considered. One possibility stems from the situation that the algorithm used here for the approximation of the vector field was not so elegant; thereby we could not obtain an unstable periodic orbit embedded in the attractor. The other possible reason could be that a new periodic solution was surely created, which was different from those embedded in the original attractor. In either case, the proposed method can successfully eliminate the fluctuations and thus would be worth being applied to actual experiments in online control.

In the actual experiments we cannot directly apply the method proposed in Section 4 to the engine system because the pressure resulting from combustion is not a directly accessible variable. A small modification is needed. Instead of the pressure, we have to find an alternative variable (for instance, the boost voltage) to directly influence the engine system, denoted by $x_{2}(\theta)$. It follows that we observe the pressure $x_{1}(\theta)$ and give the delayed feedback to the variable $x_{2}(\theta)$. In brief, the elements of $\boldsymbol{C}$ in (2) are replaced with $c_{21}=1$ and $c_{j 1}=0$ for $j=1,3 \leq j \leq m$.

\section{Acknowledgements}

We thank Ryusuke Kato and Hiroyuki Tsuzuku for providing the experimental data. One of the authors (K. M.) thanks Hans H. Diebner and Florian Grond for useful discussions. We thank Yuzuru Sato, Luis G. Moyano and Thimo Rohlf for valuable suggestions. One of the authors (I. T.) was partially supported by the Grant-in-Aid for Scientific Research on Priority Areas, No. 18019002 and No. 18047001, Grant-in-Aid for Scientific Research (B), No. 18340021, and Grantin-Aid for Exploratory Research, No. 17650056.

[3] T. Kamiński, M. Wendeker, K. Urbanowicz, and G. Litak, Chaos 14, 461 (2004).

[4] G. Litak, M. Wendeker, M. Krupa, and J. Czarnigowski, J. Vibr. Control 11, 371 (2005).

[5] M. Wendeker, J. Czarnigowski, G. Litak, and K. Szabelski, Chaos, Solitons and Fractals 18, 803 (2003). 
[6] J. B. Heywood, Internal Combustion Engine Fundamentals, McGraw Hill, New York 1988.

[7] J. C. Kantor, Science 224, 1233 (1984).

[8] C.S. Daw, M. B. Kennel, C.E. A. Finney, and F. T. Connolly, Phys. Rev. E 57, 2811 (1998).

[9] C. S. Daw, C. E. A. Finney, J. B. Green, Jr., M. B. Kennel, J. F. Thomas, and F. T. Connolly, SAE Paper No. 962086 (1996).

[10] K. Pyragas, Phys. Lett. A 170, 421 (1992).

[11] E. Ott, C. Grebogi, and J. A. Yorke, Phys. Rev. Lett. 64, 1196 (1990).

[12] H. Nakajima, Phys. Lett. A 232, 207 (1997).

[13] The four-stroke cycle, which is also called the Otto cycle, consists of the four stages: air intake, compression, combustion and exhaust.

[14] F. Takens, Lect. Notes Math. 898, 366 (1981).

[15] N. H. Packard, J. P. Crutchfield, J. D. Farmer, and R. S. Shaw, Phys. Rev. Lett. 45, 712 (1980).
[16] M. B. Kennel, R. Brown, and H. D. I. Abarbanel, Phys. Rev. A 45, 3403 (1992).

[17] M. Sano and Y. Sawada, Phys. Rev. Lett. 55, 1082 (1985).

[18] I. Tsuda and H. Fujii, J. Integrative Neurosci. 6, 309 (2007).

[19] K. Pyragas and A. Tamaševičius, Phys. Lett. A 180, 99 (1993).

[20] T. Hikihara and T. Kawagoshi, Phys. Lett. A 211, 29 (1996).

[21] S. Bielawski, D. Derozier, and P. Glorieux, Phys. Rev. E 49, R971 (1994).

[22] K. Hall, D. J. Christini, M. Tremblay, J. J. Collins, L. Glass, and J. Billette, Phys. Rev. Lett. 78, 4518 (1997).

[23] I. Tsuda, T. Tahara, and H. Iwanaga, Int. J. Bifurcation and Chaos 2, 313 (1992). 